\title{
Reacción tisular a materiales de sutura no absorbibles en piel de equinos
}

\author{
Tissue reaction to nonabsorbable suture materials in horse skin

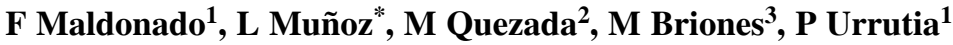 \\ ${ }^{1}$ Departamento de Ciencias Clínicas, ${ }^{2}$ Departamento de Patología y Medicina Preventiva. \\ ${ }^{3}$ Departamento de Ciencias Pecuarias, Facultad de Medicina Veterinaria, Universidad de Concepción. \\ A. Vicente Méndez 595, Chillán, Chile
}

\begin{abstract}
SUMMARY
The degree of tissue reaction of 4 nonabsorbable suture materials (linen, nylon, polymerized caprolactum and polypropylene) implanted into health skin of 8 horses was clinically and histologically evaluated. Cutaneous oedema and absence or presence of the exudation were clinically evaluated 5 and 10 days following suture material implantation. Biopsies were taken to histologically evaluate tissue reaction at 10 days. The results obtained showed that linen caused more oedema by the $5^{\text {th }}$ day, whereas the nylon and caprolactum had less oedema on the $10^{\text {th }}$ day. With respect to the presence or absence of exudation, nylon was the only material which did not show exudation by the $5^{\text {th }}$ day in any of the 8 samples, but on the $10^{\text {th }}$ day, neither the nylon, caprolactum or polypropylene showed exudation, except one horse that showed exudation for all suture materials. The histological evaluation showed that nylon and caprolactum caused less tissue reaction. In conclusion, linen was found to have a higher degree of tissue reaction in the present study, and nylon although similar to caprolactum and polypropylene in terms of tissue reaction, caused less cases of exudate than the other suture materials used.
\end{abstract}

Palabras clave: sutura, equino, piel, reacción tisular.

Key words: suture materials, horse, skin, tissue reaction.

\section{INTRODUCCION}

Una sutura es una hebra de cualquier material que se usa para aproximar un tejido y mantenerlo unido temporal o definitivamente de manera de permitir una reparación por primera intención. A través de los tiempos se han usado diversos materiales de sutura de origen, comportamiento y estructura diferentes (Maldonado y Torres 1999), los cuales se clasifican de acuerdo a su origen en naturales y sintéticos; de acuerdo al comportamiento en los tejidos en absorbibles y no absorbibles; y de acuerdo a su estructura en mono y multifilamentos (van Winkle y Hasting 1972, Stashak 1994, Niles y Williams 1999, Adams y Fessler, 2000). En general, hasta hace pocos años, la elección del material de sutura se basaba más en consideraciones personales que en el conocimiento de la interacción biológica entre el material de sutura y el tejido a suturar (van Winkle y Hasting 1972). Hoy en día los cirujanos disponen de una amplia variedad de materiales de sutura y si bien no existe un material adecuado para todas las situaciones quirúrgicas, algunos se comportan de mejor manera en

Aceptado: 09.08.2005

*Fax: (41) 207183, correo electrónico: lismunoz@udec.cl, casilla 537, Chillán. diferentes heridas, por cuanto al momento de elegir alguno de éstos se deben considerar la resistencia, tipo y condición del tejido a suturar, naturaleza, cantidad y resistencia del material de sutura, la pérdida de resistencia de la sutura y ganancia de resistencia del tejido en el tiempo (Boothe 1989, Maldonado y Torres 1999), ya que dentro de las propiedades físicas deseadas están que el material seleccionado sea resistente, permita un fácil manejo y otorgue seguridad al nudo (Rosin y Robinson 1989, Blackford y Blackford 1999), tenga mínima capilaridad, posea un calibre uniforme, no sirva como medio de desarrollo bacteriano, y de las propiedades químicas, que no tenga efecto carcinogénico, alérgico y que posea una mínima reactividad tisular (Crane 1988, Alexander 1989, Blackford y Blackford 1999, Niles y Williams 1999).

La reacción inflamatoria local asociada con el uso de materiales de sutura es debido al trauma de la inserción de la aguja y las propiedades físicas y químicas del material de sutura (van Winkle y Hasting 1972, Wood 1984). El paso de cualquier aguja y material de sutura es suficiente para provocar una inflamación aséptica, la cual dura aproximadamente cinco días y con formación de una costra microscópica por 10 a 15 días (Madsen 1953, Wood 1984). La duración e intensidad de la reacción inflamatoria varía principalmente de acuerdo al material usado, ya que éste actúa como cuerpo extraño que indu- 
ce y prolonga la reacción tisular (van Winkle y Hasting 1972, Varma y col 1981), además de la técnica quirúrgica y el tejido en el cual la sutura es implantada (Varma y col 1981, Wood 1984). La inflamación provoca aumento de la friabilidad del tejido, con lo que disminuye la tensión de la línea de sutura, demorando la cicatrización y aumentando la probabilidad de infección (Edlich y col 1973, Varma y col 1981, Wood 1984, Blackford y Blackford 1999).

Aun cuando los materiales disponibles están bien caracterizados y ha sido estudiado su comportamiento en humanos y en animales, no existen muchos estudios comparativos en equinos referentes a sus propiedades y comportamiento, por lo que se seleccionaron de acuerdo a nuestras observaciones en terreno los tres materiales de sutura más comúnmente usados en piel de equinos como son: lino, nylon y caprolactam polimerizado, y un cuarto: el polipropileno, sindicado por Blackford y Blackford (1999) como el mejor material de sutura disponible para piel en equinos.

\section{MATERIAL Y METODOS}

Se utilizaron ocho caballos mestizos de ambos sexos, de entre 16 y 22 años, clínicamente sanos, los que fueron tranquilizados con maleato de acepromazina (Acepril®, Troy Ilium), 0,05-0,1 mg/kg, vía endovenosa. Posteriormente se les rasuró un área de 10 por 10 centímetros en la parte dorsoanterior de la tabla del cuello del lado izquierdo. Esta área fue lavada y luego desinfectada con alcohol yodado al 2\%, a continuación el área fue subdividida en 4 cuadrantes (figura 1), y en la parte central de cada uno de ellos se midió el grosor del pliegue cutáneo con un pie de metro. Posteriormente se realizó una infiltración lineal en sentido horizontal con clorhidrato de lidocaína al 2\% (Lidocaína 2\% L.Ch. ${ }^{\circledR}$, Laboratorio Chile) $2 \mathrm{ml}$, vía subcutánea, para anestesiar cada área. Luego, en cada una de éstas se hicieron 3 puntos de sutura simples en piel a 1 centímetro de distancia uno de otro, para lo cual se usó nylon (Ethilon ${ }^{\circledR}$, Ethicon) 1 USP en el cuadrante superior izquierdo, lino $\mathrm{N}^{\circ} 20$ en el superior derecho; polipropileno (Prolene ${ }^{\circledR}$, Ethicon) 1 USP en el inferior izquierdo y caprolactam polimerizado (Vetafil Bengen $\AA$, WDT) 1 USP en el inferior derecho, para todos los materiales de sutura se utilizó una aguja $18 \mathrm{G}$ y se hicieron 4 nudos. Todos los puntos de sutura los hizo la misma persona. Posteriormente las áreas de sutura fueron desinfectadas con povidona yodada al $1 \%$ diariamente hasta el día 5 post sutura y luego cada 48 horas. El grosor del pliegue cutáneo del punto central fue medido los días 5 y 10 post sutura, con la finalidad de determinar el aumento de grosor del pliegue cutáneo (edema), y también se observó la presencia o ausencia de exudado. El décimo día, luego de retirar los puntos y anestesiar localmente cada cuadrante se tomó una muestra de piel de 1 centí-

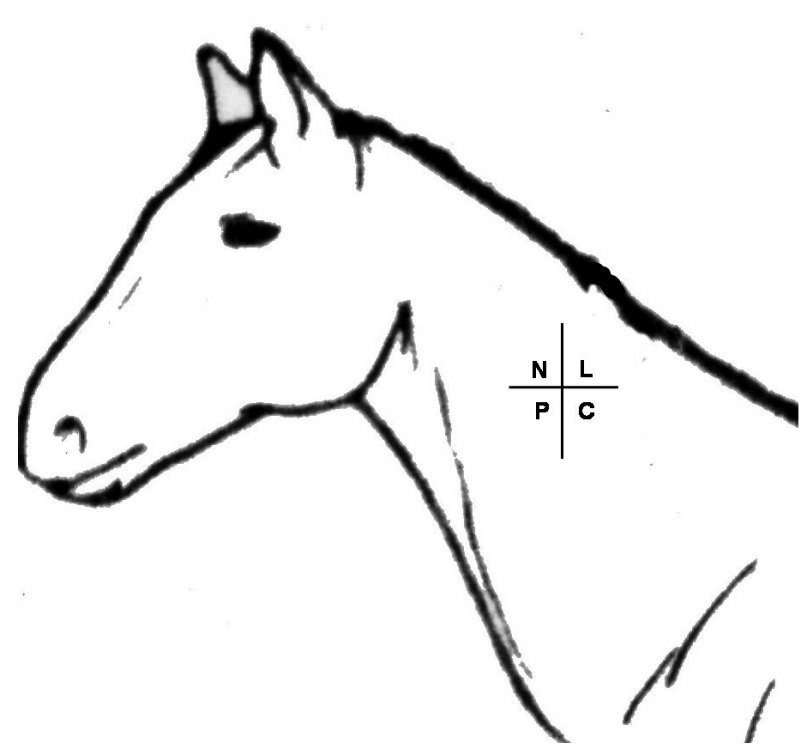

Figura 1. Esquema del lado izquierdo del cuello que señala los cuadrantes utilizados para los diferentes materiales de sutura: nylon $(\mathrm{N})$, caprolactam $(\mathrm{C})$, polipropileno $(\mathrm{P})$ y lino (L).

Diagram of the left side of the neck showing the quadrants used for the different suture materials: nylon $(\mathrm{N})$, caprolactum $(\mathrm{C})$, polypropylene (P) and linen (L).

metro cuadrado en cada uno de ellos y uno de piel intacta obtenida del punto de convergencia de las cuatro zonas de sutura y a $5 \mathrm{~cm}$ de éstas, utilizando esta biopsia como control. Las muestras de piel fueron fijadas en formalina tamponada al $10 \%$ y procesadas en el Laboratorio de Histopatología de la Facultad de Medicina Veterinaria de la Universidad de Concepción, siguiendo un protocolo estándar (Luna 1968), el que consistió en deshidratar e incluir las muestras en parafina y la realización de cortes histológicos de $4 \mu$ de espesor utilizando un micrótomo de rotación. Finalmente las secciones de tejidos fueron teñidas con hematoxilina y eosina y observadas al microscopio óptico para su evaluación. La intensidad de reacción tisular frente a los diferentes elementos de sutura utilizados fue clasificada en 4 grados:

- Grado 1: sin lesión inflamatoria, sin infiltración leucocitaria.

- Grado 2: lesión inflamatoria leve con edema y escasos leucocitos.

- Grado 3: lesión inflamatoria leucocitaria perivascular.

- Grado 4: lesión inflamatoria leucocitaria extendida y difusa en la zona analizada.

Análisis estadístico. Para el análisis de los datos se usó el programa computacional SPSS versión 8.0 para Windows (SPSS Inc, Chicago IL, USA). Para evaluar el grosor del pliegue cutáneo fueron calculados los 
parámetros poblacionales básicos (media aritmética, rango y desviación estándar), utilizándose análisis de varianza de un factor y comparaciones de promedios mediante la prueba paramétrica de Student Newman Keuls. Para evaluar la presencia o ausencia de exudado se utilizó la prueba no paramétrica de chi cuadrado y para evaluar el grado de reacción tisular se utilizó la prueba no paramétrica de Kruskal-Wallis (Daniel 1996).

\section{RESULTADOS}

El grosor del pliegue cutáneo promedio de los ocho caballos utilizados, previo al implante de los puntos de sutura, fue de 3,2 $\mathrm{mm}$ con un rango de 2,5 a $4 \mathrm{~mm}$. El promedio del grosor cutáneo en milímetros, con su respectivo rango y desviación estándar para los días 5 y 10 post sutura, se muestra en el cuadro 1.

Los resultados mostrados en el cuadro 1 permiten establecer que al $5^{\circ}$ día post sutura hubo un aumento del grosor del pliegue cutáneo (edema) para todos los materiales de sutura utilizados, siendo mayor este aumento para el lino $(\mathrm{P}<0,05)$ al compararlo con los otros tres materiales de sutura utilizados, cuyas diferencias no son estadísticamente significativas, y que si bien al $10^{\circ}$ día post sutura el promedio del grosor del pliegue cutáneo disminuyó en todos los materiales de sutura, el edema fue mayor como respuesta al lino y polipropileno $(\mathrm{P}<0,05)$ comparado con los otros dos materiales de sutura utilizados, cuyas diferencias no son estadísticamente significativas.

Los resultados referentes a la presencia o ausencia de exudado en los puntos realizados con distintos materiales de sutura muestran que tanto el día 5 como el día 10 post sutura existen diferencias significativas $(\mathrm{P}<0,05)$ entre el lino y los otros tres materiales sutura (cuadro 2). Sin embargo, cabe destacar que en un caballo se detectó presencia de exudado en los cuatro materiales de sutura el día 10.

Cuadro 1. Promedio, rango y desviación estándar (DE) del edema cutáneo provocado por los materiales de sutura utilizados al día 5 y 10 post sutura.

Mean, range and standard deviation of the cutaneous oedema for suture materials at 5 and 10 days post suture.

\begin{tabular}{lcccccccc}
\hline & \multicolumn{3}{c}{ Día 5 } & & \multicolumn{3}{c}{ Día 10 } \\
\cline { 2 - 3 } \cline { 7 - 9 } Material & $\begin{array}{c}\text { Promedio } \\
(\mathrm{mm})\end{array}$ & $\begin{array}{c}\text { Rango } \\
(\mathrm{mm})\end{array}$ & $\begin{array}{c}\mathrm{DE} \\
(\mathrm{mm})\end{array}$ & $\begin{array}{c}\text { Promedio } \\
(\mathrm{mm})\end{array}$ & $\begin{array}{c}\text { Rango } \\
(\mathrm{mm})\end{array}$ & $\begin{array}{c}\mathrm{DE} \\
(\mathrm{mm})\end{array}$ \\
\hline Lino & $8.13^{\mathrm{a}}$ & $6-10$ & 1.458 & & $7.63^{\mathrm{a}}$ & $6-11$ & 1.923 \\
Nylon & $5.50^{\mathrm{b}}$ & $4-7$ & 1.926 & & $5.00^{\mathrm{b}}$ & $4-7$ & 1.069 \\
Caprolactam & $6.25^{\mathrm{b}}$ & $5-8$ & 0.886 & & $5.38^{\mathrm{b}}$ & $4-7$ & 0.916 \\
Polipropileno & $6.12^{\mathrm{b}}$ & $4-9$ & 1.727 & & $6.00^{\mathrm{a}}$ & $4-8$ & 1.309 \\
\hline
\end{tabular}

n: 8

a: $\mathrm{P}<0,05$ vs $\mathrm{b}$
Cuadro 2. Caballos con presencia o ausencia de exudado por los materiales de sutura los días 5 y 10 post sutura.

Presence or absence of exudate to the suture materials in horses at 5 and 10 days post suture.

\begin{tabular}{|c|c|c|c|c|c|c|c|c|}
\hline \multirow[b]{2}{*}{ Exudado } & \multicolumn{2}{|c|}{$\begin{array}{l}\text { Lino } \\
\text { (días) }\end{array}$} & \multicolumn{2}{|c|}{$\begin{array}{l}\text { Nylon } \\
\text { (días) }\end{array}$} & \multicolumn{2}{|c|}{$\begin{array}{c}\text { Caprolactam } \\
\text { (días) }\end{array}$} & \multicolumn{2}{|c|}{$\begin{array}{l}\text { Polipropileno } \\
\text { (días) }\end{array}$} \\
\hline & $5^{a}$ & $10^{\mathrm{a}}$ & $5^{\mathrm{b}}$ & $10^{\mathrm{b}}$ & $5^{b}$ & $10^{\mathrm{b}}$ & $5^{b}$ & $10^{\mathrm{b}}$ \\
\hline & 2 & & 8 & & 4 & 7 & 6 & 7 \\
\hline Presente & 6 & 5 & - & 1 & 4 & 1 & 2 & 1 \\
\hline
\end{tabular}

$\mathrm{n}: 8$

a: $\mathrm{P}<0,05$ vs b

Cuadro 3. Grado de reacción tisular provocado por los materiales de sutura al día 10 post sutura.

Degree of tissue reaction for suture materials at 10 days post suture.

\begin{tabular}{|c|c|c|c|c|c|}
\hline $\begin{array}{c}\text { Grado de reacción } \\
\text { tisular }\end{array}$ & $\operatorname{Lino}^{\mathrm{a}}$ & Nylon ${ }^{b}$ & Caprolactam $^{\mathrm{b}}$ & Polipropileno ${ }^{b}$ & Control \\
\hline 1 & - & - & - & - & 8 \\
\hline 2 & - & 2 & 2 & 2 & - \\
\hline 3 & 3 & 5 & 5 & 4 & - \\
\hline 4 & 5 & 1 & 1 & 2 & - \\
\hline
\end{tabular}

n: 8

a: $\mathrm{P}<0,05$ vs $b$

Finalmente el grado de reacción tisular a los diferentes materiales de sutura el día 10 se muestra en el cuadro 3 .

Los resultados obtenidos permiten establecer que existen diferentes grados de reacción tisular, lo cual es estadísticamente significativo $(\mathrm{P}<0,05)$ entre el lino y los otros tres materiales de sutura.

\section{DISCUSION}

El primer parámetro medido fue edema resultante del implante de los materiales de sutura, el cual es definido como el acúmulo anormal de fluidos procedentes de los vasos en los espacios tisulares y que forma parte de la reacción inflamatoria (Kitt 1985). En cambio, la presencia de exudado es una manifestación de infección local (Corman y col 1981).

Haciendo un análisis individual de las propiedades de los materiales de sutura utilizados, podemos encontrar explicación a los resultados obtenidos. El nylon al ser un monofilamento sintético compuesto de poliamidas (Mansen 1953, van Winkle y Hasting 1972), de superficie no capilar (Campbell y Marks 1985, “Anónimo” 1992, Turner y Wayne 1989), suave, de textura y calibre uniforme (Leonard 1972, Hickman y Walker 1976, Turner y Wayne 1989), biológicamente inerte y que debido a que sus productos de degradación son potentes agentes 
antibacterianos (van Winkle y Hasting 1972, Blackford y Blackford 1999), ejerce un mínimo efecto como cuerpo extraño y por lo tanto la reacción inflamatoria local es mínima (Varma y col 1981, Campbell y Bailey 1992, Niles y Williams 1999), como ha sido ya determinado en estudios realizados en humanos por Getzen y Jansen (1966) y Postlethwait y col (1975). Por otro lado, el caprolactam es un multifilamento sintético (Robertson 1990, Alsted y col 2002) compuesto por poliamidas lisas y recubierto por una vaina protectora (Robertson 1990) que le da una apariencia de monofilamento, además su estructura se asemeja a la albúmina, por lo que se incorpora completamente al tejido sin provocar irritación (Turner y Wayne 1989, Stashak 1994, “Anónimo” 1995), pero que de acuerdo a lo señalado por Blackford y Blackford (1999) provoca una reacción tisular intermedia y que según Robertson (1990), al ser usado en heridas contaminadas, tiende a formar abscesos o senos que finalmente resultan en un drenaje purulento crónico. Por otra parte, el polipropileno, es un monofilamento sintético, ideal para suturar piel, ya que es prácticamente inerte en los tejidos (van Winkle y Hasting 1972, Corman y col 1981, Campbell y Marks 1985, Blackford y Blackford 1999, Niles y Williams 1999), por lo que provoca en ellos una reacción mínima (Varma y col 1981, "Anónimo" 1992). Finalmente, el lino es un multifilamento natural, que provoca una mayor reacción tisular y que además ejercería un mayor efecto como cuerpo extraño al ser implantado en piel (Stashak 1994) y que al permanecer más de 3 días aumenta la reacción inflamatoria y retrasa la cicatrización (Brunius y Ahren 1969, citado por van Winkle y Hasting 1972), por lo que debe ser evitado en heridas contaminadas (Postlethwait 1970), aun cuando Hickman y Walker (1976) lo consideraban como de escasa reacción tisular.

En estudios comparativos de diferentes autores se observan algunos patrones constantes como también resultados discrepantes. En general, los materiales de sutura sintéticos provocan una reacción tisular menor que los de origen natural (Postlethwait 1970, Postlethwait y col 1975, Varma y col 1981), por lo que de acuerdo a lo recomendado por van Winkle y Hasting (1972) deben ser removidos tempranamente para evitar la infección. También existe consenso respecto a que los multifilamentos causan mayor reacción tisular (Postlethwait 1959, Lily y col 1969 citado por van Winkle y Hasting 1972) e infección que los monofilamentos (van Winkle y Hasting 1972, Varma y col 1981, Trostle y Hendrickson 1995), ya que al ser un multifilamento permite que retenga fluidos entre sus fibras y por lo tanto lo hace más susceptible a causar infección, lo cual es más evidente en el lino que además tiene una mayor capacidad de absorción que el caprolactam, lo que evidentemente genera una mayor respuesta inflamatoria y por lo tanto una exudación (Trotter 1989), ambos patrones son coincidentes con los resultados del presente estudio. Tam- bién existen varios estudios comparativos entre el nylon y el polipropileno, algunos macroscópicos, como los realizados en piel de humanos por Madsen (1953), van Winkle y Hasting (1972), o histológicos, realizados por Varma y col (1981) en heridas cutáneas de perro, por Lily y col (1969 citado por van Winkle y Hasting 1972) en mucosa oral de perro y por de Nardo y col (1996) en mucosa oral de gatos, todos quienes señalan que no existen diferencias en la reacción tisular entre estos dos materiales de sutura; en cambio De Nardo y col (1996) en este mismo estudio y al hacer una apreciación visual determinaron que el polipropileno causaba una reacción inflamatoria local menor que el nylon, reconociendo en cierta forma lo subjetivo de una apreciación visual. Sin embargo, el presente estudio coincide con el realizado por Wood (1984) en línea alba de perro en que el nylon causó una reacción inflamatoria menor que el polipropileno. Además, un estudio en humanos señala que el 9,4\% de las suturas con polipropileno se infectan (Corma y col 1981).

La disminución del grosor del pliegue cutáneo el día 10 post sutura era previsible de acuerdo con lo señalado por Madsen (1953), quien indica que la inflamación local tiene un pico entre el $2^{\circ}$ y $7^{\circ}$ día de implantada la sutura, para luego disminuir y mantenerse mínima en el caso de los materiales no absorbibles, aun cuando la reacción a cuerpo extraño puede mantener la reacción inflamatoria y contribuir al desarrollo de infección en heridas (Getzen y Jansen 1966).

También es importante destacar que la biopsia de piel intacta (muestra control) no presentó reacción inflamatoria en ninguno de los ocho ejemplares, lo que demuestra que el efecto inflamatorio de los materiales de sutura usados fue local, no se extendió la reacción a las áreas vecinas.

Con los resultados obtenidos se puede concluir que el lino no es recomendable para suturar piel de equinos, debido a que por ser un multifilamento de origen natural provoca una mayor reacción tisular e infección de los puntos de sutura, lo que retarda la cicatrización, y que de los materiales de sutura sintéticos el nylon, si bien es similar al caprolactam y polipropileno respecto a la reacción tisular, causó una menor cantidad de equinos con presencia de exudado que los otros materiales utilizados.

\section{RESUMEN}

Se evaluó clínica e histológicamente el grado de reacción tisular frente a cuatro materiales de sutura no absorbibles (lino, nylon, caprolactam polimerizado y polipropileno), implantados en la piel de 8 equinos. Se determinó clínicamente el edema provocado y la ausencia o presencia de exudado a los 5 y 10 días de haber sido implantados los materiales de sutura, además al $10^{\circ}$ día se realizó una biopsia para determinar histológicamente el grado de reacción tisular. Los resultados obtenidos mostraron que el lino causó un mayor edema al día 
5 , mientras que al día 10 el nylon y caprolactam provocaron un menor de edema. Con respecto a la presencia o ausencia de exudado, al día 5 la sutura de nylon fue la única que no presentó exudado en ninguno de los 8 ejemplares, sin embargo, al día 10, salvo un ejemplar que presentó exudado en todos los materiales, el nylon, caprolactam y polipropileno no presentaron exudado. En cuanto a la evaluación histológica tanto el nylon como el caprolactam mostraron menor grado de reacción tisular. En conclusión, el lino resultó tener el mayor grado de reacción tisular durante el presente estudio, y el nylon si bien es similar al caprolactam y polipropileno respecto a la reacción tisular, causó una menor cantidad de equinos con presencia de exudado que los otros materiales utilizados.

\section{AGRADECIMIENTOS}

Al personal (de enfermeros de ganado) del Regimiento de Caballería Blindada $\mathrm{N}^{\circ} 7$ "Guías" por la cooperación prestada en este estudio.

\section{REFERENCIAS}

Adams SB, JF Fessler. 2000. Atlas of equine surgery. WB Saunders Company, Philadelphia.

Alexander AH. 1989. Técnicas quirúrgicas en animales y temas de terapéutica quirúrgica. Interamericana Mc Graw Hill, México DF.

Alsted H, J Jensen, L Davies, H Pedersen, R Wagner. 2002. Kruuse Veterinary Equipment. Marselev.

"Anónimo". 1992. Manual de cierre de heridas suturas. Ethicon Inc, México DF.

“Anónimo". 1995. Vademecum Veterinario. Hoechst Roussel Vet. Santiago de Chile.

Blackford LW, JT Blackford. 1999. Suture materials and patterns. En: Auer, JA, Stick JA (eds). Equine surgery. Pp 91-103. WB Saunders, Philadelphia.

Boothe HW. 1989. Suture materials and tissue adhesives. En: Slatter DH (ed). Textbook of small Animal Surgery. Pp 334344. WB Saunders, Philadelphia.

Campbell EJ, JV Bailey. 1992. Mechanical properties of suture materials in vitro and after in vivo implantation in horses. Vet Surg 21, 355-361.

Campbell JR, A Marks. 1985. Suture material and suturing techniques. In Pract 7, 72-75.

Corman ML, MC Veindenheimer, JA Coller. 1981. Controlled clinical trial of three suture materials for abdominal wall closure after bowel operations. Am J Surg 141, 510-513.

Crane SW. 1988. Guía de elección de la sutura. En: Betts CW, Stephen WC (eds). Manual de terapéutica quirúrgica de los pequeños animales. Pp 253-259. Salvat Editores, Barcelona.

Daniel W. 1996. Bioestadística. Bases para el análisis de las ciencias de la salud. Editorial Limusa SA, México DF.

De Nardo GA, NO Brow, R Trenka-Benhin, S Manfra Maretta. 1996. Comparison of seven different suture materials in the feline oral cavity. J Am Anim Hosp Assoc 32, 164-172.
Edlich RF, PA Panek, GT Rodeheaver, VG Turnbull, LD Kurtz, MT Edgerton. 1973. Physical and chemical configuration of sutures in the development of surgical infection. Ann Surg 177, 679-688.

Getzen LA, GA Jansen. 1966. Correlation between allergy to suture material and postoperative wound infections. Surg 60, 824-826.

Hickmann J, RG Walker. 1976. Atlas de cirugía veterinaria. Compañía Editorial Continental, México DF.

Kitt T. 1985. Tratado de anatomía patológica general. Editorial Labor, Barcelona.

Leonard EP. 1972. Cirugía de los pequeños animales. Editorial Científico Médica, Barcelona.

Luna LG. 1968. Manual of histologic staining methods of the Armed Forces Institute of Pathology. Mc Graw-Hill Book, New York.

Maldonado R, Torres P. 1999. Introducción a la cirugía. Bases biológicas y principios básicos. Editorial Facultad Ciencias Biológicas, Universidad de Concepción, Concepción.

Madsen ET. 1953. An experimental and clinical evaluation of surgical suture material. Surg Ginecol Obstet 97, 73-80.

Niles J, J Williams. 1999. Suture materials and patterns. In Pract 21, 308-322.

Postlethwait RW. 1970. Long-term comparative study of nonabsorbable sutures. Ann Surg 171, 892-898.

Postlethwait RW, DA Willigan, AW Ulin. 1975. Human tissue reaction to sutures. Ann Surg 181, 144-150.

Postlethwait RW, JF Schauble, ML Dillon, J Morgan. 1959. Wound healing II, an evaluation of surgical suture material. Surg Ginecol Obstet 108, 555-566

Robertson JT. 1990. Abdominal closure. En: White NA (ed). The equine acute abdomen. Pp 293-307. Lea \& Febiger, Philadelphia.

Rosin E, GM Robinson. 1989. Knot security of suture materials. Vet Surg 18, 269-273.

Stashak TS. 1994. Manejo de las heridas en equinos. Editorial Intermédica, Buenos Aires.

Trostle SS, DA Hendrickson. 1995. Suture sinus formation following closure of ventral midline incisions with polypropylene in three horses. J Am Vet Med Assoc 207, 742-745.

Trotter GW. 1989. Techniques of wound closure. Vet Clin North Am: Equine Pract 5, 499-511.

Turner AS, Wayne C. 1989. Techniques in large animal surgery. Lea \& Febiger, Philadelphia.

Van Winkle W, JC Hasting. 1972. Considerations in the choice of suture material for various tissues. Surg Ginecol Obstet 135, 113-126.

Varma S, LW Johnson, HL Ferguson, WV Lumb. 1981. Tissue reaction to suture materials in infected surgical wounds: a histopathologic evaluation. Am J Vet Res 42, 563-570.

Wood DS. 1984. Tissue reaction to nonabsorbable suture materials in the canine linea alba: a histological evaluation. J Am Anim Hosp Assoc 20, 39-44. 